\title{
BMJ Open Barriers and facilitators experienced by patients, carers and healthcare professionals when managing symptoms in infants, children and young people at end-of-life: a mixed methods systematic review protocol
}

To cite: Greenfield K, Holley S, Schoth DE, et al. Barriers and facilitators experienced by patients, carers and healthcare professionals when managing symptoms in infants, children and young people at end-of-life: a mixed methods systematic review protocol. BMJ Open 2019;9:e030566. doi:10.1136/ bmjopen-2019-030566

- Prepublication history and additional material for this paper are available online. To view these files, please visit the journal online (http://dx.doi. org/10.1136/bmjopen-2018030566).

Received 20 March 2019 Revised 12 June 2019 Accepted 21 June 2019

Check for updates

(c) Author(s) (or their employer(s)) 2019. Re-use permitted under CC BY-NC. No commercial re-use. See rights and permissions. Published by BMJ.

For numbered affiliations see end of article.

Correspondence to Professor Christina Liossi; cliossi@soton.ac.uk

\section{ABSTRACT}

Introduction This protocol describes the objective and methods of a systematic review of barriers and facilitators experienced by patients, carers and healthcare professionals when managing symptoms in infants, children and young people (ICYP) at end-of-life.

Methods and analysis The Cochrane Library, PROSPERO, CINAHL, MEDLINE, PsycINFO, Web of Science Core Collection, ProQuest Dissertations \& Theses Database, Evidence Search and OpenGrey will be electronically searched. Reference screening of relevant articles and inquiries to researchers in the field will be undertaken. Studies will be selected if they apply qualitative, quantitative or mixed-methods designs to explore barriers and facilitators experienced by patients, carers and healthcare professionals when managing symptoms in ICYP at end-of-life. Articles will be screened by title and abstract by one reviewer with a second reviewer assessing $10 \%$ of the articles. Both reviewers will read and screen all remaining potentially relevant articles. For included articles, one reviewer will extract study characteristics and one will check this. Both reviewers will undertake independent quality assessments of included studies using established and appropriate checklists including The Critical Appraisal Skills Programme Qualitative Checklist; The evaluative criteria of credibility, transferability, dependability and confirmability; The Quality Assessment Tool for Quantitative Studies, and The Mixed Methods Appraisal Tool. Data synthesis methods will be decided after data extraction and assessment.

Ethics and dissemination This review will inform our understanding of symptom management in ICYP at endof-life. The findings will be reported in a peer-reviewed journal and presented at conferences. The study raises no ethical issues.

PROSPERO registration number CRD42019124797

\section{INTRODUCTION}

Approximately 40000 infants, children and young people (ICYP) are living with a
Strengths and limitations of this study

- This systematic review can give us a greater understanding of symptom management in paediatric palliative care, highlighted as a research priority by the National Institute for Health and Care Excellence, and could inform the design of evidence-based interventions to support more effective medicine management.

- The systematic review will follow robust guidelines and the quality of included articles will be assessed using validated tools.

- The heterogeneity of the included studies, which may use qualitative, quantitative or mixed-methods approaches, could limit the overall data synthesis.

life-threatening or life-limiting condition in England. ${ }^{1}$ These include congenital anomalies, cancer and neurological, haematological, respiratory, genitourinary, perinatal, metabolic, circulatory and gastrointestinal conditions. There were nearly 3000 child deaths due to medical conditions in England in 2017, of which over 2350 were due to a known life-limiting condition or neonatal death. $^{2}$

ICYP's palliative care needs often differ from those of adults, and the diversity of conditions in this population means that practitioners must manage a wide range of complex symptoms. ${ }^{3}$ A particular challenge is managing continuous 'background' pain as well as bouts of severe, sudden-onset 'breakthrough pain', both of which are common in ICYP with a terminal illness ${ }^{4}$ and are known to be underassessed and undertreated. ${ }^{5}$ 
Family carers play a vital role in supporting ICYP with a terminal illness, allowing patients to be cared for and die at home where possible. However, there is little research on carers' experiences of administering medicines for symptom relief to ICYP receiving palliative care. Managing symptoms such as pain is potentially difficult for carers of children at home. They may lack the necessary skills and confidence required to balance symptom relief and side effects while fear of errors can lead to insufficient or inappropriate doses of analgesics. Families will move ICYP away from their preferred place of care if symptoms, including pain, are not managed effectively. ${ }^{6}$

Community nurses and doctors may also lack the skills and experience required to support carers. A systematic review found that GPs experience anxiety regarding their competency to deliver appropriate palliative care ${ }^{7}$ while healthcare support workers providing end-of-life care in the community require training in palliative care to cope with emotionally demanding situations. ${ }^{8}$

The recent National Institute for Health and Care Excellence (NICE) guideline ${ }^{9}$ is based on evidence from 20 systematic reviews investigating different aspects of planning and management of end-of-life care for ICYP with life-limiting conditions. These include reviews on what information is perceived as helpful and what social and practical support is effective for ICYP and their caregivers. The findings indicate that timely, honest and consistent information that meets individuals' needs (eg, developmentally appropriate for patients) is beneficial, including information about access to services, community and medical resources. One study also found that parents wanted information on how to use equipment that a child/young person required. ${ }^{10}$ However, symptom management was not identified as a major theme in these reviews. ${ }^{9}$

Four other reviews looked at the effectiveness of pharmacological and non-pharmacological interventions for pain management, agitation, respiratory distress and seizures. ${ }^{9}$ Only the pain management review found any studies that met the inclusion criteria and all of these involved pharmacological interventions only.

Although these reviews provide essential guidance in managing end-of-life care for ICYP, to our knowledge, no systematic review has examined the barriers and facilitators to symptom management in ICYP at end-oflife for healthcare professionals, caregivers and patients. NICE highlights pain management in palliative care as a research priority ${ }^{9}$ and a greater understanding of this could inform the design of evidence-based interventions to support more effective medicine management.

\section{Objectives}

The main objective of this systematic review is to identify and synthesise the existing literature that explores the barriers and facilitators experienced by children and young people themselves and their carers and healthcare professionals when managing symptoms in ICYP at end-of-life.

\section{METHODS}

This protocol follows the Preferred Reporting Items for Systematic Review and Meta-Analysis Protocols $(\text { PRISMA-P })^{11}$ guidelines (see online supplementary file 1-PRISMA-P checklist) and is registered (ID CRD42019124797) ${ }^{12}$ on PROSPERO, an international register of systematic reviews. ${ }^{13}$ Any changes to the protocol will be recorded on PROSPERO.

The reporting of the systematic review will be informed by the Centre for Reviews and Dissemination ${ }^{14}$ and the Cochrane Qualitative Research Methods Group guidelines $^{15}$ and will follow the Enhancing Transparency in Reporting the Synthesis of Qualitative Research $(\text { ENTREQ) })^{16}$ and the PRISMA statements ${ }^{11}$ for reporting systematic reviews (see online supplementary file 2). In the case of sections applicable to qualitative systematic reviews that are included in PRISMA, but are not covered by ENTREQ, these will also be reported.

\section{Eligibility criteria}

The criteria outlined below will be used for study selection. Following the recommendations of the Cochrane Qualitative and Implementation Methods Group Guidance,${ }^{15}$ we have used STARLITE (Sampling Strategy, Type of study, Approaches, Range of years, Limits, Inclusions and exclusions, Terms used, Electronic sources ${ }^{17}$ ) to report our search methods.

\section{Sampling strategy}

This review will consider all studies carried out worldwide that involve carers, healthcare professionals or patients' views on symptom management in ICYP up to the age of 24 years at end-of-life care. A cut-off age of 24 years will be used since this corresponds to adolescent growth and current understandings of this stage in life. ${ }^{18}$

\section{Type of study}

The review will consider qualitative, quantitative and mixed-method studies including questionnaires, surveys, interviews, focus groups, case studies and observations. Trials, cohort and intervention studies that assess barriers and facilitators to symptom management will all be considered.

\section{Approaches}

In addition to searching electronic databases, the search strategy will include hand searching of reference lists of identified eligible studies. Finally, active researchers in the field who have contributed to this literature will be contacted.

\section{Range of years}

Studies published from the inception of each database will be included.

Limits

Articles written in any language other than English will not be searched due to a lack of funding for adequate 
translation, along with masters theses, conference abstracts and reviews.

\section{Inclusions}

Studies reporting barriers and facilitators experienced by carers, healthcare professionals and the patients themselves, when managing symptoms in ICYP with terminal illnesses receiving palliative care and/or at end-of-life will be included. All definitions of 'end-of-life' will be included since there are a wide variety of definitions and there is a paucity of research in ICYP symptom management in this area. Data on carers, healthcare professionals and patients' views, attitudes, opinions, perceptions, beliefs or feelings will be included.

\section{Exclusions}

Studies that focus only on the effectiveness of pharmacological treatments for symptom management will be excluded.

\section{Searches}

\section{Electronic sources}

The Cochrane Library and PROSPERO will be searched initially to check for any existing systematic reviews on this topic. As recommended by the Cochrane Qualitative and Implementation Methods Group, ${ }^{15}$ CINAHL (Cumulative Index of Nursing and Allied Health Literature) via Ebsco and Ovid MEDLINE will be searched, as well as PsycINFO via Ebsco and the Web of Science Core Collection. To identify any additional unpublished work, the ProQuest Dissertations \& Theses Database, Evidence Search and OpenGrey will also be searched. The search strategy will include hand searching of reference lists of eligible studies for additional records. All searches will be run during February 2019.

\section{Search terms used}

A search strategy was developed based on the 'Managing Pain' search strategy used in the NICE guideline 'End-oflife Care for Infants, Children and Young people with Life-limiting Conditions: Planning and Management (NG61)'.9 The strategy incorporated search terms in four blocks: (1) 'Patient Population'; (2) 'Caregivers and Patients'; (3) 'End-of-life'; and (4) 'Pain and Symptoms.' Additional searches used in the Palliative Care Search Filter $^{19}$ were also incorporated into Block 3 for each database.

Combinations of keywords, text words, Medical Subject Headings $(\mathrm{MeSH})$ and other terms relevant to the four blocks were selected for each database to optimise the search sensitivity and specificity. The search strategy was piloted and adapted for each database. A professional healthcare research librarian assisted in the development of the strategy. Please see online supplementary file 3 for the full search strategy for each database.

\section{Data management}

All records and data will be saved to Endnote X8. ${ }^{20}$ This software will be used to identify potential duplicates. The researchers will check this and remove all confirmed duplicated articles.

\section{Selection process}

Articles will be screened by title and abstract by one reviewer $(\mathrm{KG})$ with a second reviewer $(\mathrm{SH})$ assessing $10 \%$ of the articles, randomly selected. At this stage, articles will be judged as either (1) 'not relevant' or (2) 'potentially relevant'. Both reviewers will read and screen all remaining potentially relevant articles. The reviewers will independently apply the criteria at all stages of the selection process. Intercoder agreement will be evaluated using Cohen's kappa coefficient. A minimum kappa value of 0.75 will be taken to represent high agreement. ${ }^{21}$

The full text of all remaining potentially relevant articles will then be obtained. If the relevance of a study cannot be ascertained from the abstract, then the full article will be obtained. The full articles will be read by two reviewers independently ( $\mathrm{KG}$ and $\mathrm{SH}$ ) to make the final decision about whether they should be chosen for inclusion in the review. A third reviewer (CL) will resolve any uncertainties. Additional information will be sought from authors if necessary at the stage of full-text assessment.

\section{Data collection process and items}

The following information will be extracted into a piloted data collection form for all included studies: study aims; patient population (infant/child/adolescent); participant population (patient/caregiver/healthcare professional); inclusion and exclusion criteria; sample size; recruitment; design; intervention and comparator group (where applicable); date and duration of data collection; setting; country; data collection; analysis methods; data describing the participants' views/experiences of barriers and facilitators to symptom management. For qualitative data, the authors' interpretations (presented through themes and categories) will represent these data. ${ }^{22} \mathrm{KG}$ will extract this information and $\mathrm{SH}$ will check it, with any disagreements resolved through discussion with CL.

\section{Quality assessment (including risk of bias)}

A quality appraisal of included studies will be conducted independently by two reviewers ( $\mathrm{KG}$ and $\mathrm{SH}$ ). Disagreements will be resolved by discussion between $\mathrm{KG}$ and $\mathrm{SH}$, with CL if required.

Three checklists will be used depending on each study's design. These were chosen since they are all validated and have been used in published systematic reviews within healthcare research. For each study type, Cohen's kappa coefficient will be used to measure inter-rater agreement between the two reviewers. A minimum kappa value of 0.75 will be taken to represent high agreement with disagreements resolved via discussion with CL.

As recommended by the Cochrane Qualitative and Implementation Methods Group, ${ }^{15}$ we will not calculate total quality scores across domains since domains of quality are not equal. Instead, $\mathrm{KG}, \mathrm{SH}$ and $\mathrm{CL}$ will determine how each study's methodological limitations affect 
confidence in the findings via discussion. We will not exclude studies based on poor quality but will record and highlight methodological issues.

Qualitative studies will be quality appraised using the Critical Appraisal Skills Programme for Qualitative Studies (CASP) ${ }^{23}$ CASP assesses clarity of research aims, research design, recruitment methods, data collection, relationships between participants and researchers, ethical issues, analyses, description of findings and valuableness of the research. It is comprised of nine closed questions (eg, 'Was there a clear statement of the aims of the research?' Yes/Can't tell/No) and one open-ended question ('How valuable is the research?'). For each question, there is the option to add comments to explain the reasoning for each rating. Currently, CASP is the most frequently used qualitative research synthesis tool in the Cochrane Library and WHO guideline research ${ }^{24}$ and has been used in similar systematic reviews assessing barriers and facilitators within healthcare research ${ }^{25} 26$ However, because the CASP tool does not address aspects of the research validity and can favour papers that are less insightful as long as they comply with 'expectations of research practice', ${ }^{27}$ in addition, the evaluative criteria of credibility, transferability, dependability and confirmability ${ }^{28}$ will be applied. Included studies will be assessed as to whether they apply the techniques suggested for ensuring study quality according to Guba and Lincoln's ${ }^{28}$ criteria, that is, prolonged engagement, persistent observation, peer review, triangulation, negative case analysis, referential adequacy and member checking to ensure credibility; thick description for transferability; inquiry audit for dependability; confirmability audit, audit trail, triangulation and reflexivity to ensure confirmability. Studies will be rated as 'high quality' if they meet at least three of the four criteria, 'medium quality' if they meet two of the criteria and 'low quality' if they meet one or none.

The Quality Assessment Tool for Quantitative Studies (QATQS) will be used to assess all clinical studies with or without randomisation and control groups, including quasi-experimental and before-and-after studies. ${ }^{29}$ The QATQS is comprised of 22 closed questions and an overall rating of strong, moderate or weak in eight sections: selection bias; study design; confounders; blinding; data collection; withdrawals and dropouts; intervention integrity; analysis. It has been shown to be a valid tool for assessing quality, comparing studies and addressing threats to validity of findings. ${ }^{30}$

The Mixed Methods Appraisal Tool (V.11) will be used to assess the quality of any mixed methods studies. ${ }^{31}$ This tool consists of five closed questions assessing the research question, research design, integration of qualitative and quantitative methods, integration of qualitative and quantitative data and consideration of methodological limitations in mixed methods studies. As reported by the Cochrane Qualitative and Implementation Methods Group, this tool has been used widely in systematic reviews and has the advantage of being able to assess interdependent qualitative and quantitative elements of mixed-methods research. ${ }^{24}$

\section{Outcomes and prioritisation}

The main outcomes sought are carers', healthcare professionals' and patients' (CYP) views on the barriers and facilitators to effective symptom management in ICYP at end-of-life.

\section{Data synthesis}

Although it is unlikely that the majority of included studies will be quantitative, if this is the case, then random-effects meta-analysis will be conducted to synthesise group means and standard deviation from individual studies using Comprehensive Meta-Analysis version $3{ }^{32}$

For meta-analysis to be conducted, data must be available from two or more eligible studies reporting similar barriers or facilitators. The studies must report the number of participants reporting that barrier/facilitator and the total number of valid participant responses for that survey item. A random-effects model will be used for all analyses since; unlike a fixed-effects model, this can be used when statistical heterogeneity $\left(\mathrm{I}^{2}\right)$ is present in the results of the included studies. ${ }^{33}$ Where evidence of statistically significant heterogeneity is present, sensitivity analyses will be conducted where possible to verify the robustness of the study conclusions, assessing the impact of methodological quality, study design, sample size and the potential effects of missing data. We will use funnel plots to detect potential reporting biases and small-study effects where data are available from 10 or more studies. ${ }^{34}$

If the included studies are all qualitative or a combination of quantitative and qualitative, there are several approaches that could be taken for data synthesis. Some of the most commonly used methods to synthesise qualitative health research include thematic analysis, ${ }^{35}$ grounded theory ${ }^{36}$ and meta-ethnography. ${ }^{37}{ }^{38}$ However, there is no consensus on the best approach, which will depend on the type and number of included studies ${ }^{39}$ and the form and nature of the research question. ${ }^{38}$ As such, we will make a final decision on the most appropriate method after selecting and quality assessing the included articles, as recommended by the Cochrane Qualitative and Implementation Methods Group. ${ }^{39}$

We will first analyse and synthesise data related to the experience of patients, care providers and healthcare professionals separately before deciding whether it is appropriate to aggregate data between these groups. These data will likely include themes, concepts and categories of information. If data are relatively 'thin', then we will consider using thematic synthesis to undertake line-by-line coding and development of descriptive and analytic themes. If the included articles include sufficient 'thick' data (eg, details about the context and background of the studies and participants ${ }^{40}$ ), we will consider a more interpretative approach such as meta-ethnography. ${ }^{37}$ This method goes beyond aggregating data to generate new interpretations of the findings. 
As recommended by the Cochrane Qualitative and Implementation Methods Group, ${ }^{24}$ the GRADE-CERQual (Confidence in the Evidence from Reviews of Qualitative research ${ }^{41}$ ) will be used to summarise our confidence in synthesised qualitative findings (eg, in the themes that we identify). The CERQual is made up of four key components, that is, methodological limitations of included studies, coherence of the review finding, adequacy of the data contributing to a review finding and relevance of the included studies to the review question. After assessing each of the four components, overall confidence will be graded as high, moderate, low or very low. The barriers and facilitators to symptom management will be divided into overarching themes for each group (patients, healthcare professionals, carers), and presented in a matrix along with our CERQual assessment of confidence in the evidence of each theme and an explanation of this assessment.

The GRADE guidelines ${ }^{42}$ will be used to appraise the quality of any quantitative findings. The GRADE guidelines include four elements for which quantitative findings will be rated against: risk of bias ('Study limitations'), inconsistency, indirectness, imprecision, publication bias.

\section{Patient and public involvement}

Consultation with young people, parents and healthcare professionals has been used to determine their perception of the barriers and facilitators they experience when managing symptoms in ICYP at end-of-life. It is based on their perspectives that this systematic review was deemed to be timely and crucial to conduct to inform further research work. Moreover, patient and public involvement (PPI) is represented in the authorship (MJ) of this manuscript. MJ is the PPI representative at the UK National Institute for Health Research (NIHR) Pain and Palliative Care Clinical Studies Group-Children. MJ is a parent of four children, one of whom died from T-cell acute lymphoblastic leukaemia at the age of 12. She has supported many families of children with cancer including those receiving palliative and end-of-life care.

\section{DISCUSSION}

This systematic review will be the first to synthesise and report barriers and facilitators experienced by patients, carers and healthcare professionals when managing symptoms in ICYP at end-of-life. The dearth and heterogeneity of the included studies, which may use qualitative, quantitative or mixed-methods approaches, could limit the overall data synthesis we are able to conduct. As we expect there to be a lack of suitable studies, they will not be excluded on the basis of quality, which may limit the confidence in our findings. The review findings will be used to inform our ongoing work to develop a structured educational tool to support carers and healthcare professionals to administer pain and symptom relief to ICYP at the end-of-life.

\section{ETHICS AND DISSEMINATION}

As this is a systematic review of published literature, ethical approval will not be sought. We will publish the protocol and our findings in peer-reviewed journals aimed at paediatric palliative care clinicians and researchers as well as health commissioners. We will present our work at the growing numbers of national and international meetings focused on paediatric palliative care and pain.

\section{Author affiliations}

${ }^{1}$ Department of Psychology, University of Southampton, Southampton, UK

${ }^{2}$ Helen \& Douglas House Hospices, Oxford, UK

${ }^{3}$ Department of Anaesthesia and Pain Medicine, Great Ormond Street Hospital for Children NHS Foundation Trust, London, UK

${ }^{4}$ The Louis Dundas Centre, Great Ormond Street Hospital for Children NHS Foundation Trust, London, UK

${ }^{5}$ Specialist Palliative Care Team, Alder Hey Children's Hospital, Liverpool, UK

${ }^{6}$ Rainbows Hospice for Children and Young People, Loughborough, UK

${ }^{7}$ Patient Representative c/o Public Health and Primary Care, University of

Cambridge, Cambridge, UK

${ }^{8}$ School of Pharmacy, University College London, London, UK

Acknowledgements We would like to thank Dr Liz Jamieson for her comments on an earlier version of this protocol.

Contributors EH, RH, MJ, IW and CL conceived the idea, planned and designed the study protocol. KG, SH, DES planned the data extraction and statistical analysis and wrote the first draft; JB, LB and SJ provided critical insights. All authors have approved and contributed to the final written manuscript.

Funding This study was supported by Great Ormond Street Children's Charity and SPARKS (Grant number: V5118).

Competing interests None declared.

Patient consent for publication Not required.

Provenance and peer review Not commissioned; externally peer reviewed.

Open access This is an open access article distributed in accordance with the Creative Commons Attribution Non Commercial (CC BY-NC 4.0) license, which permits others to distribute, remix, adapt, build upon this work non-commercially, and license their derivative works on different terms, provided the original work is properly cited, appropriate credit is given, any changes made indicated, and the use is non-commercial. See: http://creativecommons.org/licenses/by-nc/4.0/.

\section{REFERENCES}

1. Fraser LK, Miller M, Hain R, et al. Rising national prevalence of life-limiting conditions in children in England. Pediatrics 2012;129:e923-9.

2. Petrou S, Fraser J, Sidebotham P. Child death in high-income countries. The Lancet 2014;384:831-3.

3. Himelstein BP, Hilden JM, Boldt AM, et al. Pediatric palliative care. $N$ Engl J Med 2004;350:1752-62.

4. Oostendorp LJ, Rajapakse D, Kelly P, et al. Documentation of breakthrough pain in narrative clinical records of children with life-limiting conditions: Feasibility of a retrospective review. J Child Health Care 2018:136749351880731.

5. Friedrichsdorf SJ, Postier A. Management of breakthrough pain in children with cancer. J Pain Res 2014;7:117-23.

6. Brombley K. Understanding the Factors that Influence Parents as they Plan and Manage their Child's End of Life Care Rome: Foundazione Maruzza. 2016 http://www.childrenpalliativeca recongress.org/congress-2016/wp-content/uploads/2016/02/ PROGRAMMA_2016_REV_11_bassa.pdf.

7. Mitchell GK. How well do general practitioners deliver palliative care? A systematic review. Palliat Med 2002;16:457-64.

8. Herber OR, Johnston BM. The role of healthcare support workers in providing palliative and end-of-life care in the community: a systematic literature review. Health Soc Care Community 2013;21:225-35.

9. National Institute for Health and Care Excellence. End of life care for infants children and young people: planning and management (NICE Guideline NG61): National Institute for Health and Care Excellence, 2016.

10. Hunt A, Coad J, West E, et al. Together for Short Lives. The Big Study for Life-limited Children and their Families - Final research report. 2013 http://clok.uclan.ac.uk/8951/2/TfSL_The_Big_Study_ Final_Research_Report_WEB_.pdf. 
11. Moher D, Liberati A, Tetzlaff J, et al. PRISMA Group. Preferred reporting items for systematic reviews and meta-analyses: the PRISMA statement. Ann Intern Med 2009;151:264-9.

12. Greenfield K, Holley S, Schoth D, et al. Systematic review of the barriers and facilitators experienced by patients, carers and healthcare professionals when managing symptoms in infants, children and young people at end-of-life CRD42019124797: PROSPERO. 2019 http://www.crd.york.ac.uk/PROSPERO/display_ record.php?ID=CRD42019124797.

13. Booth A, Clarke M, Dooley G, et al. The nuts and bolts of PROSPERO: an international prospective register of systematic reviews. Syst Rev 2012;1:2.

14. NHS Centre for Reviews and Dissemination. Undertaking systematic reviews of research on effectiveness: CRD's guidance for those carrying out or commissioning reviews: York: University of York, 2001.

15. Harris JL, Booth A, Cargo M, et al. Cochrane Qualitative and Implementation Methods Group guidance series-paper 2: methods for question formulation, searching, and protocol development for qualitative evidence synthesis. J Clin Epidemiol 2018;97:39-48.

16. Tong A, Flemming K, Mclnnes $\mathrm{E}$, et al. Enhancing transparency in reporting the synthesis of qualitative research: ENTREQ. BMC Med Res Methodol 2012;12:181.

17. Booth A. "Brimful of STARLITE": toward standards for reporting literature searches. J Med Libr Assoc 2006;94:421-9.

18. Sawyer SM, Azzopardi PS, Wickremarathne D, et al. The age of adolescence. Lancet Child Adolesc Health 2018;2:223-8.

19. Sladek R, Tieman J, Fazekas BS, et al. Development of a subject search filter to find information relevant to palliative care in the general medical literature. J Med Libr Assoc 2006;94:394.

20. Endnote X8 Referencing Software [program]. New York, NY, 2017.

21. Landis JR, Koch GG. The measurement of observer agreement for categorical data. Biometrics 1977;33:159.

22. Munabi-Babigumira S, Glenton C, Lewin S, et al. Factors that influence the provision of intrapartum and postnatal care by skilled birth attendants in low- and middle-income countries: a qualitative evidence synthesis. Cochrane Database Syst Rev 2017;11:CD011558.

23. CASP. CASP Qualitative Checklist 2018. 2019 https://casp-uk.net/ wp-content/uploads/2018/03/CASP-Qualitative-Checklist-2018 fillable_form.pdf (accessed 18.01.2019).

24. Noyes J, Booth A, Flemming K, et al. Cochrane Qualitative and Implementation Methods Group guidance series-paper 3 : methods for assessing methodological limitations, data extraction and synthesis, and confidence in synthesized qualitative findings. $J$ Clin Epidemiol 2018;97:49-58.

25. Panagiotopoulou N, Ghuman N, Sandher R, et al. Barriers and facilitators towards fertility preservation care for cancer patients: a meta-synthesis. Eur J Cancer Care 2018;27:e12428.
26. Graham-Rowe E, Lorencatto F, Lawrenson JG, et al. Barriers and enablers to diabetic retinopathy screening attendance: Protocol for a systematic review. Syst Rev 2016;5:134.

27. Dixon-Woods M, Sutton A, Shaw R, et al. Appraising qualitative research for inclusion in systematic reviews: a quantitative and qualitative comparison of three methods. J Health Serv Res Policy 2007;12:42-7.

28. Guba EG, Lincoln YS. Naturalistic inquiry. Newbury Park, CA: Sage, 1985.

29. Thomas H. Quality assessment tool for quantitative studies. Effective Public Health Practice Project. Toronto: McMaster University, 2012.

30. Armijo-Olivo S, Stiles CR, Hagen NA, et al. Assessment of study quality for systematic reviews: a comparison of the Cochrane Collaboration Risk of Bias Tool and the Effective Public Health Practice Project Quality Assessment Tool: methodological research. J Eval Clin Pract 2012;18:12-18.

31. Pluye P, Gagnon MP, Griffiths F, et al. A scoring system for appraising mixed methods research, and concomitantly appraising qualitative, quantitative and mixed methods primary studies in Mixed Studies Reviews. Int J Nurs Stud 2009;46:529-46.

32. Biostat. comprehensive meta-analysis (Version 3)[Computer software] [program], 2015

33. Haidich AB. Meta-analysis in medical research. Hippokratia 2010;14(Suppl 1):29.

34. Higgins JP, Green S. Cochrane handbook for systematic reviews of interventions: The Cochrane Collaboration. 2011 www.handbook. cochrane.org.

35. Thomas J, Harden A. Methods for the thematic synthesis of qualitative research in systematic reviews. BMC Med Res Methodol 2008;8:45.

36. Glaser BG, Strauss AL, Strutzel E. The discovery of grounded theory; strategies for qualitative research. Nurs Res 1968;17:364.

37. Noblit GW, Hare RD, Hare R. Meta-ethnography: Synthesizing qualitative studies: Sage, 1988

38. Dixon-Woods M, Agarwal S, Jones D, et al. Synthesising qualitative and quantitative evidence: a review of possible methods. $J$ Health Serv Res Policy 2005;10:45-53.

39. Booth A, Noyes J, Flemming K, et al. Guidance on choosing qualitative evidence synthesis methods for use in health technology assessments of complex interventions. Integrate-HTA 2016.

40. Ponterotto JG. Brief note on the origins, evolution, and meaning of the qualitative research concept thick description. The Qualitative Report 2006;11:538-49.

41. Lewin $\mathrm{S}$, Glenton $\mathrm{C}$, Munthe-Kaas $\mathrm{H}$, et al. Using qualitative evidence in decision making for health and social interventions: an approach to assess confidence in findings from qualitative evidence syntheses (GRADE-CERQual). PLoS Med 2015;12:e1001895.

42. Guyatt GH, Oxman AD, Schünemann HJ, et al. GRADE guidelines: a new series of articles in the Journal of Clinical Epidemiology. J Clin Epidemiol 2011;64:380-2. 